\title{
Investigations of Au Segregation in AuNi Alloys by the Line Shape Analysis of Photoelectron Spectra
}

\author{
B. $\operatorname{LESIAK}^{a, *}$, A. BilińsKi ${ }^{a}$ AND A. JóŹWIK ${ }^{b, c}$ \\ ${ }^{a}$ Institute of Physical Chemistry, Polish Academy of Sciences \\ Kasprzaka 44/52, 01-224 Warszawa, Poland \\ ${ }^{b}$ Institute of Biocybernetics and Biomedical Engineering \\ Polish Academy of Sciences \\ Księcia Trojdena 4, 02-109 Warszawa, Poland \\ ${ }^{c}$ Computer Engineering Department, Technical University of Łódź \\ al. Politechniki 11, 90-924 Eódź, Poland
}

(Received November 2, 2005; revised version March 6, 2006)

The surface segregation of $\mathrm{Au}$ in Au5Ni95 polycrystalline alloy is studied applying the line shape analysis, known as the fuzzy $k$-nearest neighbour rule, to the selected X-ray photoelectron and X-ray induced Auger spectra. Each line, characterised by the kinetic electron energy exhibited in the particular transition, samples the depth described by the mean escape depth depending on the inelastic mean free path of electron in the investigated material and the geometry of the measurement. The following electron transitions in the electron kinetic energy range 216.6-1486.6 eV are analysed: $\mathrm{Au} N_{5} N_{67} V$, Ni $2 p$, Ni $L_{2} M_{23} M_{45}-\mathrm{Ni} L_{3} M_{45} M_{45}$, Au $4 d$, Au $4 f$ and the valence band transitions (overlapping $\mathrm{Au} 5 d 6 s$ and $\mathrm{Ni} 3 d 4 s$ transitions). The results of the quantitative analysis using the fuzzy $k$-nearest neighbour rule are compared to the results of quantitative analysis by the multiline approach which applies the $\mathrm{Au} 4 f$ and $\mathrm{Ni} 2 p$ photoelectron transitions. Both methods demonstrate $\mathrm{Au}$ surface segregation starting at the temperature above $200^{\circ} \mathrm{C}$. The line shape analysis was shown to be applicable for quantification of the surface region with possibility of investigating the in-depth non-uniform concentration profiles.

PACS numbers: $61.82 . \mathrm{Bg}, 68.35 . \mathrm{Dv}, 79.60 .-\mathrm{i}$

\section{Introduction}

The binary AuNi alloys, ultrathin metal film growth on metal substrate, i.e. $\mathrm{Au} / \mathrm{Ni}$ and $\mathrm{Ni} / \mathrm{Au}$, as well as their interfaces, the surface composition, the

*corresponding author; e-mail: blo@ichf.edu.pl 
electronic structure, and the surface and interface processes have already been investigated in numerous experimental and theoretical studies [1-12]. These systems have been frequently used for numerous commercial applications. In recent development in thin film technology and multilayer structures, there has been an emphasis on bimetallic surfaces produced by metal on metal heteroepitaxy. Recent advances in experimental epitaxial growth techniques enhance the capability to grow artificially structured materials. Such heteroepitaxial metal growth offers the opportunity to create metastable structures with novel physical and chemical properties. The processes of segregation, the diffusion of constituents, the temperature and the composition dependent segregation profiles have attracted attention of researchers [1-4].

The experimental studies on AuNi systems surface are based on electron spectroscopy methods, like the X-ray photoelectron spectroscopy (XPS), Auger electron spectroscopy (AES) [6, 7], the elastic peak electron spectroscopy (EPES) [7, 9-12], impact collision ion scattering spectroscopy [5] and the X-ray diffraction analysis (XRD) [8]. For quantitative analysis with the XPS and AES spectroscopy methods, as well as for determining the mean escape depth (MED) [13-15] of photoelectrons and Auger electrons, the electron transport parameter, i.e. the inelastic mean free path (IMFP) [16] of electrons is required. The MED, $D$, is defined as the average depth normal to the surface from which the electron escapes without energy loss [13]:

$$
D=\frac{\int_{0}^{\infty} z \phi(z, \alpha) \mathrm{d} z}{\int_{0}^{\infty} \phi(z, \alpha) \mathrm{d} z},
$$

where $\alpha$ is the electron emission angle measured with respect to the surface normal, $z$ is the depth from the surface and $\phi(z, \alpha)$ is the emission depth distribution function (DDF). The value of DDF is defined as the probability that the electron leaving the surface in a given direction originates from a defined depth normal to the surface, whereas the information depth (ID) is the maximum depth, normal to the surface, from which signal information is obtained [13]. If elastic scattering of detected electron is negligible, the roughly estimated value of MED is given by $[14,15]$ :

$$
D=\lambda \cos \alpha,
$$

where $\lambda$ is the value of the IMFP, depending on the electron kinetic energy and the material [16]. The value of MED depends then on the geometry of measurement and the type of electron transition. Accounting for electron elastic scattering, the value of MED is smaller than the value evaluated from Eq. (2).

Quantitative methods for analysing the surface composition are based on the measured intensities of selected photoelectron and Auger electron transitions and involve correction factors $[17,18]$. The most efficient quantitative approach for multicomponent systems accounting for the XPS transitions in the wide range of electron kinetic energies and the correction factors is the multiline (ML) ap- 
proach [18]. In this method, a new expression for the universal energy dependence of the inelastic mean free path was used, the statistical analysis has been modified, and a database with physical constants such as photoionisation cross-sections, asymmetry parameters, binding energies, etc. was added in order to avoid errors of polynomial approximations. For the $\mathrm{AuCu}$ alloys, the average deviation from the bulk surface composition was found to be $\pm 3.2 \%$. Alternative approach for both, qualitative and quantitative approach is based on the line shape analysis of the XPS, AES/XAES (X-ray AES) [19-24] and EPES [20, 25, 26] spectra using the pattern recognition methods. Such approach was applied previously for analysing the XPS and AES spectra recorded for selected complex systems, i.e. carbons exhibiting various texture, undoped and doped polymers, polycrystalline silicon [19], CuPd alloys [20, 21], CoPd alloys [20, 22], sodium silicate glasses [23] and aluminium sodium silicate glasses [24]. The processes of surface segregation in alloys [20-22] and glasses, as well as the processes of glass surface decomposition due to electron irradiation [24, 25] were studied. Due to properties of the elastic peak involving the electrons elastically backscattered [27] and quasi-elastically backscattered [28] on the atoms of the surface, the line shape analysis of the EPES spectra recorded for the multicomponent systems was found to be applicable for investigating the surface properties, the atomic composition and the processes at the surfaces $[20,25,26]$. The pattern recognition method applied to the EPES spectra has also been found convenient for analysing the sample texture, the average grain size and the surface segregation of $\mathrm{Pd}[20,25]$ and $\mathrm{Au}[20,26]$ in Co30Pd70, Co50Pd50 and Au2Ni98 alloys, respectively. The advantage of applying the pattern recognition method to the line shapes of the EPES spectra is possibility of analysing the selected and top surface information depths ranging from the first monolayers to several $\AA$.

In the present work, the quantitative analysis of the XPS/XAES spectra based on the line shape analysis using the pattern recognition method is applied for investigating the Au segregation and the in-depth profile concentrations in annealed Au5Ni95 alloy.

\section{Pattern recognition method}

The pattern recognition methods deal with classification of objects described by a set of features [29]. The most powerful approaches of object classification are the standard $k$-nearest neighbour $(k \mathrm{NN})$ rule [30] and the fuzzy $k$-nearest neighbour $(\mathrm{f} k \mathrm{NN})$ rule $[31,32]$. Both these versions of the $k \mathrm{NN}$ rule are based on a distance measure between the objects in the feature space. The method requires the representation of the spectra in a form of an $n$-dimensional vector, $\boldsymbol{x}\left(x_{1}, x_{2}, \ldots, x_{n}\right)$, where the components of the vector, $x_{i}, i=1,2, \ldots, n$, refer to the intensities of the spectra recorded at the particular $n$-th channels. The $n$-dimensional vector represented as a point in the $n$-dimensional space is called an object. The components of the vector are called the features of the object. The 
set of spectra (objects) recorded at the sample representing the same chemical state of atom or the same composition creates an $n$-dimensional cluster of points called a class. The set of $n c$ classes representing spectra recorded from samples of different chemical state of atoms or chemical composition is called a reference set, i.e. the reference set consists of $n c$ classes and $m$ vectors.

The application of the pattern recognition method is performed in the following stages:

(i) selecting and recording the classes of objects for creating the reference set which is treated as the database for classification and identification purpose;

(ii) constructing the decision rule (i.e. classifier) using information contained in the reference set, which promises the smallest misclassification rate; the classifier allows to assign the class membership to the new objects from outside of the reference set;

(iii) classifying the objects of uncertain membership using the developed classifier.

The $k \mathrm{NN}$ rule [30] assigns the classified object, i.e. a vector or a point in the feature space, to the same class as the majority of its $k$-nearest objects in the reference set. The classifier quality criterion, depending on the number of the $k$-nearest neighbours, is called the error rate, $E_{k}$, defined as

$$
E_{k}=\Delta m / m,
$$

where $\Delta m$ is the number of the misclassified objects within the reference set and $m$ is the total number of the objects in the reference set. The error rate, $E_{k}$, is calculated for all possible values of $k$ using the "leave one out" method [29]. The "leave one out" method consists in classifying each of $m$ objects from the reference set by the $k \mathrm{NN}$ rule derived from the remaining $m-1$ objects. The minimum value of the error rate, $E_{k}$, which is a function of $k$ is selected as a classification and identification quality measure.

The decision of $k \mathrm{NN}$ [30] rule is described as a membership vector $\boldsymbol{\nu}\left(\nu_{1}, \nu_{2}, \nu_{3}, \ldots, \nu_{n c}\right)$, where $\nu_{i}=0$ or 1 . The $\mathrm{f} k \mathrm{NN}[31,32]$ decision is described as the fuzzy membership vector $\boldsymbol{\nu}\left(\nu_{1}, \nu_{2}, \nu_{3}, \ldots, \nu_{n c}\right)$, where $\nu_{i}$ is the $i$-th class contribution among $n c$ classes, and $\nu_{i}=k_{i} / k$, where $k_{i}$ is the number of the objects from the $i$-th class among the $k$-nearest neighbours of the classified object in the reference set.

The value of the error rate can be decreased using the feature selection procedure. It consists in rejecting the redundant features and preserving only the most informative features. The objects can be described by features which are less informative or not relevant to the considered classification problem. These redundant features can spoil the classification performance. This phenomenon is called the "dimensionality paradox" [33] - more information about the recognized objects leads to lower percentage of the correctly classified objects. To find the optimum feature subset of the whole available set of $n$ features it is neces- 
sary to review all possible feature combinations, to calculate the misclassification rate (error rate) for each of the reviewed feature subset and to select the feature combination that offers the smallest error rate. Reviewing of all possible feature combinations for the $n$ features is computationally very costly. For this reason the backward feature selection procedure can be applied. It starts with calculation of the misclassification rate for all $n$ features. The $n$ feature combinations of $n-1$ features are analysed and the best subset of $n-1$ features is selected. Then, from $n-1$ feature combinations $n-2$ feature sets are selected in the next step, and the best subset of $n-2$ features is chosen. In the similar manner, sequentially the feature combinations of $n-3, n-4$, etc. features are selected, ending on one single feature set. Finally, the best reviewed feature combination is selected. The classification problem using the $k \mathrm{NN}$ rule is characterised by the reference set containing $n c$ classes, $m$ vectors described by the $n$ features and the error rate, $E_{k}$, which is a function of the $k$-nearest neighbours.

The pattern recognition method is applied to spectra represented by the intensity and energy normalised $n$-dimensional objects. For all the spectra, the intensities are normalised to the maximum value equal to 1 , whereas all the maxima are shifted to one common energy position. In such approach only the changes in the analysed line shapes are considered in calculations.

\section{Experimental}

\subsection{Sample preparation}

The AuNi alloys of nominal bulk atomic percent composition Au80Ni20, Au50Ni50, Au20Ni80, Au10Ni90 and Au5Ni95, were prepared from the Ni foil (4N, Johnson Matthey, UK) and the Au foil (4N, Mennica Państwowa, Poland) by induction melting of the weight amount components in conditions allowing to obtain homogeneous solution. All the alloys were prepared in a form of a rolled foil. The surfaces of all the samples were polished in water suspended allumina.

\subsection{X-ray photoelectron spectroscopy}

The measurements were carried out in the ESCALAB-210 X-ray photoelectron spectrometer (V.G. Scientific, UK) with a base pressure in the chamber of $10^{-9}$ Torr. The spectrometer is equipped with a dual anode $\mathrm{X}$-ray source $\left(\mathrm{Mg} K_{\alpha}, \mathrm{Al} K_{\alpha}\right)$, a high resolution energy analyser, and a high energy $\mathrm{Ar}^{+}$source.

Sample surfaces were sputter cleaned with an $\mathrm{Ar}^{+}$ion beam of energy $9 \mathrm{keV}$ and a current of 4-16 $\mu \mathrm{A}$ until the carbon and oxygen contamination were not detected in the spectra. The spectra were recorded at X-ray incidence angle of $60^{\circ}$ and detection angle of $0^{\circ}$, with respect to the surface normal. The following Al $K_{\alpha}$ induced XPS/XAES spectra were recorded at metallic $\mathrm{Au}, \mathrm{Ni}$, and five AuNi alloys: Au $N_{5} N_{67} V$, Ni $2 p$, Ni $L_{2} M_{23} M_{45}-\mathrm{Ni} L_{3} M_{45} M_{45}$, Au $4 d$, Au $4 f$ and the valence band transitions (overlapping $\mathrm{Au} 5 d 6 s$ and $\mathrm{Ni} 3 d 4 \mathrm{~s}$ transitions) in the electron kinetic energy range $216-266.6 \mathrm{eV}, 596.6-646.6 \mathrm{eV}, 826.6-876.6 \mathrm{eV}$, 
1121.6-1161.6 eV, 1386.6-1406.6 eV, and 1451.6-1491.6 eV. The XPS/XAES narrow scan spectra were recorded with the energy step size of $0.1 \mathrm{eV}$ and dwell time of $200 \mathrm{~ms}$. Each type of spectrum was recorded about twenty times.

Surface segregation of $\mathrm{Au}$ in Au5Ni95 alloy was studied in the temperature range from the room temperature $(\mathrm{RT})$ to $700^{\circ} \mathrm{C}$. The Au5Ni95 alloy was placed on the support, where the sample was annealed and its temperature was monitored with the thermocouple. The sputter cleaning, the annealing at every temperature and the XPS measurements for the Au5Ni95 alloy proceeded in situ in the UHV chamber. First, the Au5Ni95 alloy surface was sputter cleaned, then the alloy was annealed to the required temperature and finally the XPS/XAES spectra were recorded for the sample.

\section{Results}

\subsection{Quantitative XPS analysis by $M L$}

The results of the ML [18] quantitative analysis accounting for the $\mathrm{Ni} 2 p$ and $\mathrm{Au} 4 f$ transition applied to the sputter cleaned AuNi alloys and to sputter

TABLE I

Results of the XPS ML [18] approach for the sputter cleaned and annealed AuNi alloy surfaces. The values of MED are evaluated from Eq. (2). The values of IMFP in the investigated alloys are taken from the TPP-2M [16] predictive formula.

\begin{tabular}{|c|c|c|c|c|c|c|}
\hline \multirow[t]{3}{*}{ Sample } & \multirow{3}{*}{$\begin{array}{c}\text { Temperature } \\
{\left[{ }^{\circ} \mathrm{C}\right]}\end{array}$} & \multicolumn{3}{|c|}{ XPS [at.\%] } & \multicolumn{2}{|c|}{$\operatorname{MED}[\AA]$} \\
\hline & & $\mathrm{Au}$ & $\mathrm{Au}$ & $\mathrm{C}$ & \multirow{2}{*}{$\mathrm{Ni} 2 p_{3 / 2}$} & \multirow{2}{*}{$\mathrm{Au} 4 f_{7 / 2}$} \\
\hline & & without $\mathrm{C}$ & \multicolumn{2}{|c|}{ including $\mathrm{C}$} & & \\
\hline \multicolumn{5}{|l|}{$\mathrm{KE}[\mathrm{eV}]$} & 633.6 & 1402.6 \\
\hline \multicolumn{7}{|c|}{ Sputter cleaned } \\
\hline Au $80 \mathrm{Ni} 20$ & $\mathrm{RT}$ & 73 & 68 & 6 & 9.1 & 16.2 \\
\hline Au $50 \mathrm{Ni} 50$ & $\mathrm{RT}$ & 34 & 32 & 4 & 10.6 & 19.0 \\
\hline $\mathrm{Au} 20 \mathrm{Ni} 80$ & $\mathrm{RT}$ & 16 & 15 & 4 & 10.2 & 18.2 \\
\hline Au10Ni90 & $\mathrm{RT}$ & 11 & 11 & 3 & 10.5 & 18.9 \\
\hline Au5Ni95 & $\mathrm{RT}$ & 5 & 5 & 7 & 11.3 & 20.4 \\
\hline \multicolumn{7}{|c|}{ Annealed Au5Ni95 } \\
\hline & 200 & 8 & 7 & 5 & & \\
\hline & 300 & 15 & 15 & 5 & & \\
\hline & 400 & 19 & 17 & 6 & & \\
\hline & 500 & 23 & 22 & 5 & & \\
\hline & 600 & 24 & 26 & 5 & & \\
\hline & 700 & 28 & 26 & 6 & & \\
\hline
\end{tabular}


cleaned and annealed in the temperature range $200-700^{\circ} \mathrm{C}$ Au5Ni95 alloy are listed in Table I. For the sputter cleaned surfaces the $\mathrm{O}$ contamination was not detected, whereas the $\mathrm{C}$ contamination varies from 3 at.\% to 7 at.\%. For the sputter cleaned and annealed Au5Ni95 surface the $\mathrm{O}$ contamination was not detected, whereas the $\mathrm{C}$ contamination does not exceed 6 at.\%. The atomic compositions without accounting for $\mathrm{C}$ were also evaluated to be compared to the results obtained from the line shape analysis. The values of the mean escape depth related to Ni $2 p$ and $\mathrm{Au} 4 f$ photoelectron transitions considered in the ML approach are also listed in Table I.

At the applied sputtering conditions, for alloys of high Au atomic percent composition, preferential surface enrichment with $\mathrm{Ni}$ is obtained, whereas for the case of alloys of smaller Au atomic percent composition (Au10Ni90 and Au5Ni95) no preferential sputtering of components is shown (Table I). This result from different sputtering yields for $\mathrm{Ni}$ and $\mathrm{Au}$ depending on the $\mathrm{Ar}^{+}$energy and the initial nominal bulk composition [34].

The annealed Au5Ni95 alloy exhibits slow Au surface segregation starting between $200^{\circ} \mathrm{C}(8$ at. $\%$ of $\mathrm{Au})$ and $300^{\circ} \mathrm{C}(15$ at.\% of $\mathrm{Au})$, and reaching 28 at.\% of $\mathrm{Au}$ at the temperature of $700^{\circ} \mathrm{C}$ (Table I).

\subsection{Quantitative analysis of XPS/XAES line shapes by the fkNN rule}

The exemplary selected XPS/XAES sets of reference spectra recorded for $\mathrm{Au}, \mathrm{Ni}$, and $\mathrm{AuNi}$ alloys at room temperature are shown in Fig. 1a, b. The respective spectra for the identification on the basis of constructed reference sets (Fig. 1a, b) using the developed classifiers are shown in Fig. 2a, b.
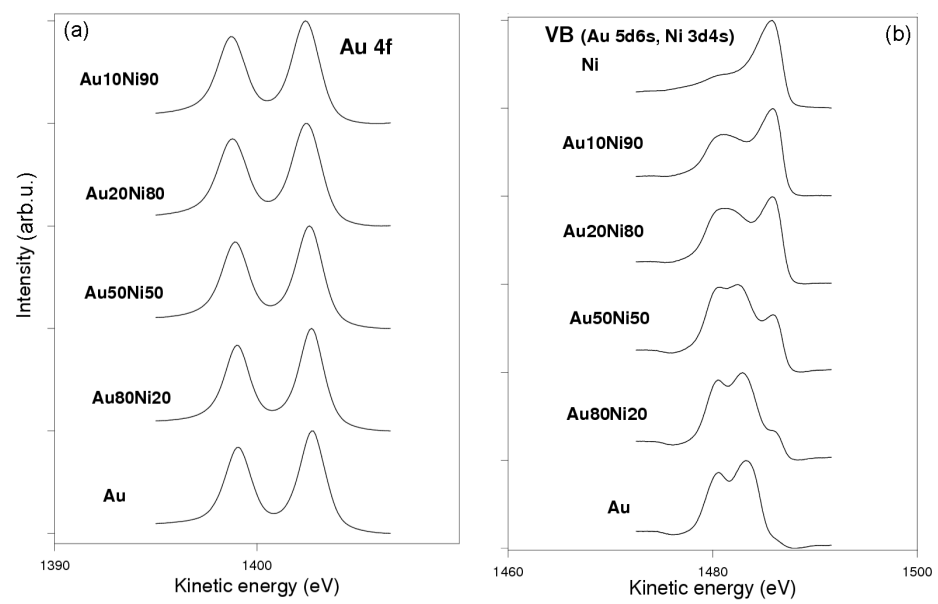

Fig. 1. The exemplary spectra recorded for the sputter cleaned Au, Ni, and AuNi alloys (Au10Ni90, Au20Ni80, Au50Ni50, Au80Ni20): (a) Au 4f, (b) valence band (overlapping $\mathrm{Au} 5 d 6 s$, Ni $3 d 4 s)$. 

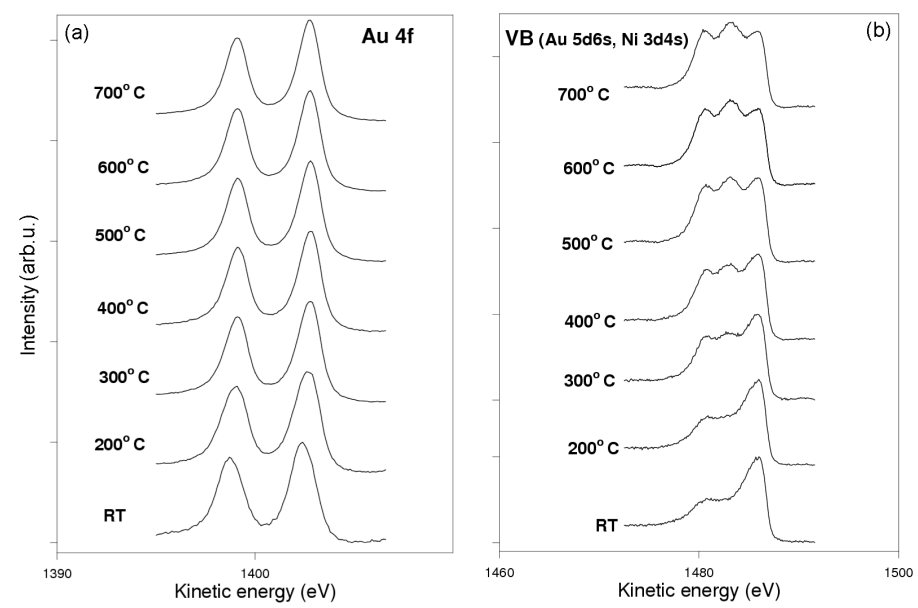

Fig. 2. The exemplary spectra recorded for the sputter cleaned and annealed Au5Ni95 alloy: (a) Au $4 f$, (b) valence band (overlapping $\mathrm{Au} 5 d 6 s$, Ni $3 d 4 s$ ).

Comparison of classifiers based on different reference sets containing the XPS/XAES spectra recorded for all considered electron transitions of various kinetic energies and mean escape depth values is given in Table II. These classifiers are based on the reference sets containing the number of classes from $n c=5$ (for $\mathrm{Au} N N V$, Ni $2 p$, Ni $L M M, \mathrm{Au} 4 d$ and $\mathrm{Au} 4 f$ transitions) to $n c=6$ (for the valence band transition). The $\mathrm{Au} N N V, \mathrm{Au} 4 d$ and $\mathrm{Au} 4 f$ transitions are recorded for $\mathrm{Au}$ and $\mathrm{AuNi}$ alloys (five samples), the $\mathrm{Ni} 2 p$ and $\mathrm{Ni} L M M$ transitions are recorded for $\mathrm{Ni}$ and $\mathrm{AuNi}$ alloys (five samples), whereas the valence band transitions are recorded for $\mathrm{Au}, \mathrm{Ni}$, and $\mathrm{AuNi}$ alloys (six samples). For obtaining the smaller value of the error rate, the selected reference sets were submitted to the feature selection procedure. This was a case for the reference sets containing the $\mathrm{Au} N N V, \mathrm{Au} 4 d, \mathrm{Au} 4 f$ and the valence band transitions. In all the cases, the error rate, estimated by the "leave one out" method, was approaching zero. Then, the results presented in Table II indicate large values of the probability of correct classification for the applied reference sets and identification of the uncertain spectra.

The quantitative results obtained by the pattern recognition line shape analysis for various XPS/XAES transitions recorded for the sputter cleaned Au5Ni95 alloy at room temperature are listed in Table III. The mean escape depth values typical of the selected electron transitions are given for comparison. At the room temperature, the in-depth $\mathrm{Au}$ composition profile averaged over the $\mathrm{Au} N N V$ Auger transition and the overlapping $\mathrm{Au}$ and $\mathrm{Ni}$ valence band transitions shows the $\mathrm{Au}$ atomic composition of 5 at.\%.

Comparison of the ML [18] quantitative results and the line shape pattern recognition method results for the annealed Au5Ni95 alloy is given in Fig. 3. The ML [18] approach indicates the Au surface enrichment above $200^{\circ} \mathrm{C}$. The line 
TABLE II

Comparison of parameters of classifiers developed on the basis of the reference sets enclosing the XPS/XAES spectra, i.e. $\mathrm{Au} N_{5} N_{67} V, \mathrm{Ni} 2 p, \mathrm{Ni} L_{2} M_{23} M_{45}-$ Ni $L_{3} M_{45} M_{45}, \mathrm{Au} 4 d$, Au $4 f$ and the valence band (overlapping $\mathrm{Au} 5 d 6 s$ and $\mathrm{Ni} 3 d 4 s$ transitions). The symbols denote: $m$ - the number of spectra, $n c$ - the number of classes, $n$ - the number of features, $k$ - the number of the $k$-nearest neighbours, $E_{k}$ - the error rate.

\begin{tabular}{l|c|c|c|c|c}
\hline \hline \multicolumn{1}{c|}{ XPS/XAES spectrum } & $m$ & $n c$ & $n$ & $k$ & $E_{k}$ \\
\hline $\mathrm{Au} N_{5} N_{67} V$ & 108 & 5 & 21 & 4 & 0.10 \\
$\mathrm{Ni} 2 p$ & 141 & 5 & 501 & 1 & 0.01 \\
$\mathrm{Ni} L_{2} M_{23} M_{45}-\mathrm{Ni} L_{3} M_{45} M_{45}$ & 135 & 5 & 501 & 3 & 0.01 \\
$\mathrm{Au} 4 d$ & 135 & 5 & 28 & 4 & 0 \\
$\mathrm{Au} 4 f$ & 139 & 5 & 30 & 12 & 0 \\
$\mathrm{VB}$ & 164 & 6 & 20 & 11 & 0
\end{tabular}

TABLE III

Comparison of quantitative results of sputter cleaned Au5Ni95 alloy at room temperature obtained by the $\mathrm{f} k \mathrm{NN}$ rule applied to the XPS/XAES spectra. The values of MED for the selected geometry of analysis and kinetic energy of the analysed electron transitions are evaluated from Eq. (2). The values of IMFP in Au5Ni95 alloy are taken from the TPP-2M [16] predictive formula.

\begin{tabular}{l|c|c|c}
\hline \hline \multirow{2}{*}{ XPS/XAES spectrum } & \multicolumn{2}{|c|}{ f $k$ NN $[$ at.\%] } & MED $[\AA]$ \\
\cline { 2 - 3 } & $\mathrm{Au}$ & $\mathrm{Ni}$ & \\
\hline $\mathrm{Au} N_{5} N_{67} V$ & 10 & 90 & 6.1 \\
$\mathrm{Ni} 2 p$ & 10 & 90 & 11.3 \\
$\mathrm{Ni} L_{2} M_{23} M_{45}-\mathrm{Ni} L_{3} M_{45} M_{45}$ & & 100 & 14.0 \\
$\mathrm{Au} 4 d$ & 20 & 80 & 17.5 \\
$\mathrm{Au} 4 f$ & 10 & 90 & 20.4 \\
$\mathrm{VB}$ & & 100 & 21.1
\end{tabular}

shape analysis based on various XPS/XAES electron transitions indicates non-linear character with the temperature, i.e. the Au surface enrichment at the top surface, and then decrease (Fig. 3). The values of atomic composition resulting from the line shape analysis averaged over the $\mathrm{Au} N N V$ Auger transition and the overlapping $\mathrm{Au}$ and $\mathrm{Ni}$ valence band transitions are shown in Fig. 3 for comparison. Due to the electron transitions applied in quantifications, both approaches, i.e. the ML and the line shape analysis accounting for averaged atomic composi- 


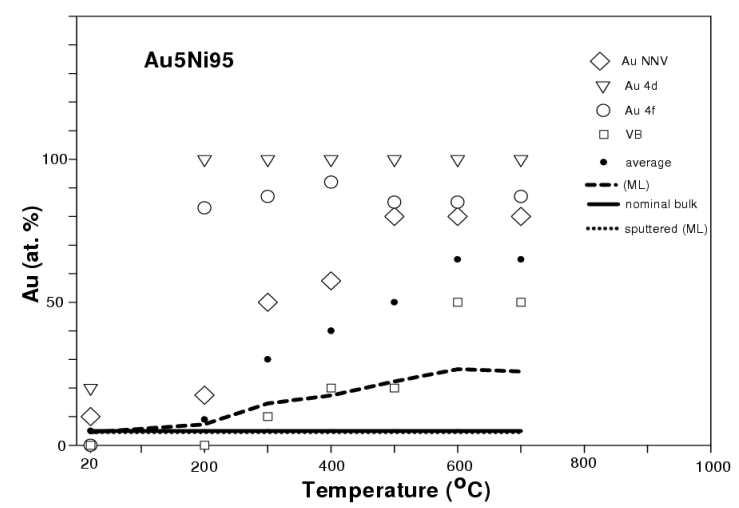

Fig. 3. Dependence of Au atomic composition in Au5Ni95 alloy with the temperature. Solid line: the nominal bulk composition. Dotted line: the results by the ML [18] approach based on $\mathrm{Au} 4 f$ and $\mathrm{Ni} 2 p$ photoelectron lines for the sputtered surface. Dashed line: the results by the ML [18] approach based on $\mathrm{Au} 4 f$ and Ni $2 p$ photoelectron lines for the annealed surface. Symbols: the results by the $\mathrm{f} k \mathrm{NN}$ rule based on different XPS/XAES transitions. $\diamond: \mathrm{Au} N N V . \nabla$ : $\mathrm{Au} 4 d$. ०: $\mathrm{Au} 4 f . \quad \square:$ valence band (overlapping $\mathrm{Au} 5 d 6 s, \mathrm{Ni} 3 d 4 s$ ). •: averaged results over $N N V$ and the valence band transitions.

tion resulting from the $N N V$ and the valence band transitions, consider similar information depths, however not exactly the same.

For the case of Au5Ni95 alloy, the Au in-depth composition changes resulting from analysing of all the selected transitions, recorded at exemplary temperature conditions, i.e. the room temperature, $300^{\circ} \mathrm{C}, 400^{\circ} \mathrm{C}, 500^{\circ} \mathrm{C}$, and $600^{\circ} \mathrm{C}$, are

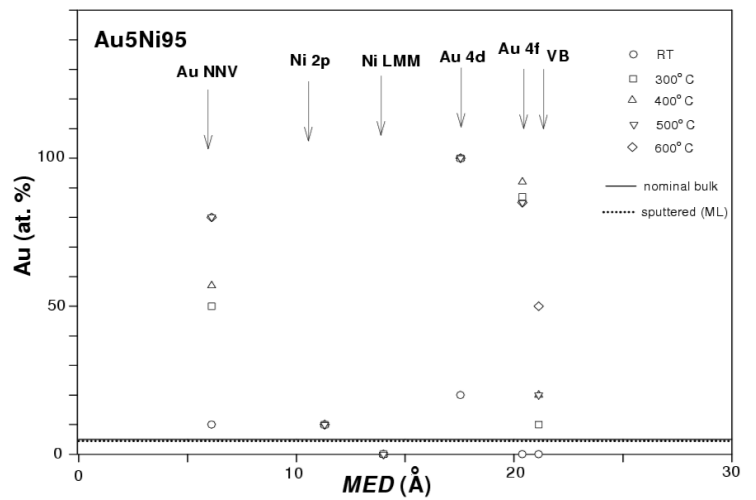

Fig. 4. Dependence of Au atomic composition in sputter cleaned and annealed Au5Ni95 alloy with the MED of electrons. Solid line: the nominal composition. Dotted line: the results by $\mathrm{ML}$ [18] approach based on $\mathrm{Au} 4 f$ and $\mathrm{Ni} 2 p$ photoelectron lines for the sputtered surface. Symbols: the results by the $f k N N$ rule based on different XPS/XAES electron transitions of various kinetic energies. $\circ$ : room temperature. $\square: 300^{\circ} \mathrm{C} . \Delta$ : $400^{\circ} \mathrm{C} . \nabla: 500^{\circ} \mathrm{C} . \diamond: 600^{\circ} \mathrm{C}$. 


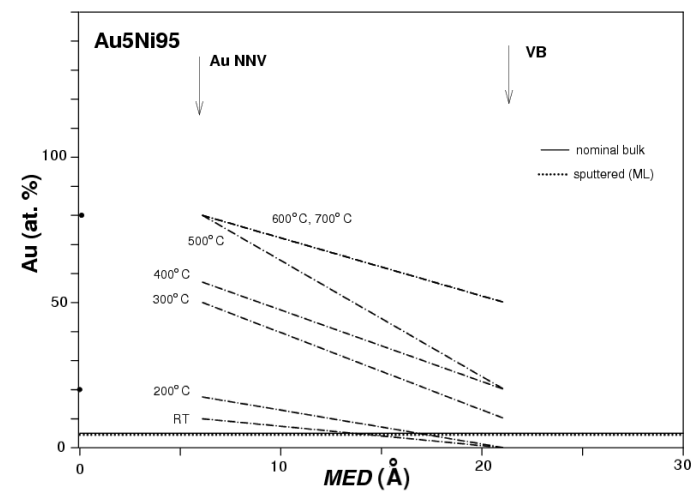

Fig. 5. Straight line approximation of the Au in-depth composition profile in the sputter cleaned and annealed Au5Ni95 alloy based on the results by the line shape analysis of the $N N V$ and the overlapping valence band transitions. Dash-dotted line: temperature dependent $\mathrm{Au}$ composition profile with the MED of electron. Solid line: the nominal bulk composition. Dotted line: the results by the ML [18] approach based on $\mathrm{Au} 4 f$ and Ni $2 p$ photoelectron lines for the sputtered surface.

compared in Fig. 4. The in-depth $\mathrm{Au}$ composition changes start between the temperature $200^{\circ} \mathrm{C}$ and $400^{\circ} \mathrm{C}$, remaining similar in the temperature range 500 $700^{\circ} \mathrm{C}$. The line shape analysis of Ni $2 p$ and Ni $L M M$ transitions do not indicate the quantitative changes due to annealing and the atomic content of $\mathrm{Au}$ remains similar between the room temperature and $700^{\circ} \mathrm{C}$ (Fig. 4). The line shape analysis of $\mathrm{Au} 4 d$ and $\mathrm{Au} 4 f$ photoelectron transitions indicates the reasonable quantitative results at the room temperature, whereas after annealing strong increase in atomic concentration occurs (Fig. 4).

Changes of the in-depth composition profile evaluated from the line shape analysis of the averaged $N N V$ and the valence band transitions in the temperature range from the room temperature to $700^{\circ} \mathrm{C}$ are shown in Fig. 5. In all the cases, the non-linear composition profile occurs, varying the shape with the temperature.

\section{Discussion and conclusions}

The results of both quantitative approaches, i.e the ML and the $\mathrm{f} k \mathrm{NN}$ rule, remain in consistency and are in agreement with the literature data. At room temperature, the investigated sputter cleaned Au5Ni95 alloy indicates Au surface enrichment (Table III, Figs. 3-5), confirmed by other authors [9, 10]. Then, after annealing the Au5Ni95 alloy indicates strong Au surface segregation (Table I, Figs. 3-5) which was previously reported experimentally [35-37] and predicted theoretically [1-4, 37]. The quantitative results obtained from the ML analysis indicate averaged Au surface enrichment without tracing the in-depth non-uniform concentration profiles which are expected to occur in Au5Ni95 alloy (Table I, Fig. 3). The results of the line shape analysis indicate the non-uniform composition 
profile after sputter cleaning (Table III, Figs. 3-5), which can be expected due to different values of sputtering yields for components [34]. After annealing, the line shape approach indicates the Au surface enrichment exhibiting the non-linear depth profile (Figs. 3-5). However, it seems that the quantitative results based on the line shape analysis are the most reliable when accounting for transitions involving electrons from outer shells, i.e. $N N V$ Auger and the valence band overlapping photoelectron transitions. As shown in Fig. 4, the quantifications using the line shape analysis of $\mathrm{Ni} 2 p$, Ni $L M M, \mathrm{Au} 4 d$ and $\mathrm{Au} 4 f$ seems to be less reliable. Generally, the line shape of inner shell electron transitions is expected to undergo changes due to chemical bond and composition variation. However, these changes may be less sensitive and less visible than the changes in the line shape involving the valence electrons. Better performance of the line shape analysis to the inner shell transitions might be expected and achieved for improved classification procedure and its parameters, e.g. the population of objects and classes in the reference set. The Au composition changes after annealing obtained from averaging the quantitative results of the $\mathrm{Au} N N V$ Auger and the valence band transitions are larger than these changes resulting from the ML approach [18] (Fig. 3).

The values of the information depth for two approaches differ slightly (Table I, Table III), however the theoretically predicted depth profile exhibits the oscillatory character with the strong Au surface enrichment at the top surface [1]. The in-depth composition profiles and their temperature dependence estimated from the straight line approximation accounting for the $N N V$ and the valence band transitions indicate $\mathrm{Au}$ surface enrichment with the shape variation in a function of the temperature (Fig. 5). This variation of the shape can be expected due to different values of diffusion coefficient for $\mathrm{Au}$ and $\mathrm{Ni}$, depending on the alloy composition and the annealing temperature [3, 4]. Similar character of segregation profile has been previously predicted by the theoretical model [1]. For AuNi alloys the Au surface segregation is observed in all the cases. For alloys of selected atomic compositions, i.e. Au5Ni95, the Au segregation exhibits the additional feature of clearly defined oscillations [1]. The in-depth non-uniform composition profile segregation in $\mathrm{CuPd}[20,21]$, CoPd [20, 22], has also been shown in the experimental studies employing the line shape analysis to the selected XPS/XAES electron transitions. Similarly, the line shape analysis of the EPES spectra was applied for quantification of $\mathrm{CoPd}[20,25]$ and $\mathrm{AuNi}[20,26]$ alloys and the in-depth non-uniform composition profiles due to $\mathrm{Pd}$ and $\mathrm{Au}$ surface segregation.

The line shape analysis has been found to be the most efficient when applied to the transition involving the electrons from the valence band, in both the Auger electron transitions and the valence band transitions. It seems that the proposed method may be perspective for application to multicomponent systems for quantitative evaluation of the overlapping valence band transitions, where the correction factors are not defined and the fitting procedures are not efficient. It may be found 
to be effective for application in the photoemission spectroscopy when investigating the very surface in-depth profiles and the interfaces in overlayer/substrate (e.g. adsorbate on the surface) and multilayer systems.

\section{Acknowledgments}

The authors (B.L., A.B., A.J.) acknowledge the support of the project KBN 4T09A 041 24. The author (B.L.) acknowledges the support of the project EU SURPHARE GMA1-2002-72057.

\section{References}

[1] B. Good, G.H. Bozzolo, P.B. Abel, Surf. Sci. 454/456, 602 (2000).

[2] M. J. H. van Dal, M.C.L.P. Pleumeekere, A.A. Kodensov, F.J.J. van Loo, J. Alloys Comp. 309, 132 (2000).

[3] R. Filipek, J. Mol. Liq. 86, 69 (2000).

[4] J. Mimkes, M. Wuttig, Thermochim. Acta 282/283, 165 (1996).

[5] K. Umezawa, S. Nakanishi, W.M. Gibson, Surf. Sci. 426, 225 (1999).

[6] A. Zafeirratos, S. Kennou, Appl. Surf. Sci. 173, 69 (2000).

[7] J. Zemek, P. Jiricek, A. Jablonski, B. Lesiak, Appl. Surf. Sci. 199, 138 (2002).

[8] T. Bigault, F. Bocquet, S. Labat, O. Thomas, H. Renevier, Appl. Surf. Sci. 188, $110(2002)$

[9] M. Krawczyk, L. Zommer, J.W. Sobczak, A. Jablonski, M. Petit, C. Robert-Goumet, B. Gruzza, Surf. Sci. 566/568, 856 (2004).

[10] M. Krawczyk, L. Zommer, J.W. Sobczak, A. Jablonski, Appl. Surf. Sci. 235, 15 (2004).

[11] J. Pavluch, L. Zommer, Y. Polyak, Z. Pekárek, A. Jablonski, B. Lesiak, T. Hrnčiř, Surf. Interface Anal. 37, 998 (2005).

[12] L. Zommer, A. Jablonski, J. Electron Spectrosc. Relat. Phenom. 150, 56 (2005).

[13] Standard E 673-01, Annual Book of ASTM Standards 2002, ASTM International, West Conshohocken, PA, 2002, Vol. 3.06, p. 755.

[14] A. Jablonski, I.S. Tilinin, C.J. Powell, Phys. Rev. B 54, 10927 (1996).

[15] A. Jablonski, C.J. Powell, Surf. Sci. 551, 106 (2004).

[16] S. Tanuma, C.J. Powell, D.R. Penn, Surf. Interface Anal. 21, 165 (1994).

[17] J.F. Moulder, W.F. Stickle, P.E. Sobol, K.D. Bomben, Handbook of X-ray Photoelectron Spectroscopy, Perkin-Elmer Co., Eden Prairie, MN, 1992.

[18] A. Jablonski, B. Lesiak, L. Zommer, M.F. Ebel, H. Ebel, Y. Fukuda, Y. Suzuki, S. Tougaard, Surf. Interface Anal. 21, 724 (1994).

[19] B. Lesiak, Vide-Sci. Technique at Appl. 52, 99 (1996).

[20] B. Lesiak, A. Jóźwik, Prace Naukowe. Elektronika 153, 79 (2005) (in Polish).

[21] B. Lesiak, A. Biliński, A. Jóźwik, Polish J. Chem. 79, 1365 (2005).

[22] B. Lesiak, A. Biliński, A. Jóźwik, Surf. Interface Anal. 37, 1143 (2005).

[23] J. Zemek, P. Jiricek, O. Gedeon, B. Lesiak, A. Jozwik, J. Non-Crystal. Solids 351, 1665 (2005). 
[24] B. Lesiak, J. Zemek, O. Gedeon, A. Jóźwik, Surf. Interface Anal. 38, 610 (2006).

[25] B. Lesiak, J. Zemek, P. Jiricek, A. Jóźwik, J. Alloys Comp., in press.

[26] B. Lesiak, J. Zemek, P. Jiricek, A. Jóźwik, Surf. Interface Anal., in press.

[27] G. Gergely, Prog. Surf. Sci. 71, 31 (2002).

[28] D. Laser, M.P. Seah, Phys. Rev. B 47, 9836 (1993).

[29] O.R. Duda, P.E. Hart, D.G. Stork, Pattern Classification, Wiley Interscience, New York 2001.

[30] E. Fix, J.L. Hodges, Discriminatory Analysis: Nonparametric Discrimination Small Sample Performance, project 21-49-004, Report Number 11, USAF School of Aviation Medicine, Randolph Field, Texas 1952, p. 280.

[31] A. Jóźwik, Pattern Recognition Lett. 1, 287 (1983).

[32] J.C. Bezdek, S.K. Chuah, D. Leep, Fuzzy Sets Systems 18, 237 (1986).

[33] R.O. Duda, P.E. Hart, Pattern Classification and Scene Analysis, Wiley, New York 1973.

[34] Y. Yamamura, H. Tawara, At. Data Nucl. Data Tables 149, 62 (1996).

[35] F.L. Williams, M. Boudart, J. Catal. 30, 438 (1973).

[36] J.J. Burton, C.R. Helms, R.S. Polizzotti, J. Vac. Sci. Technol. 13, 204 (1976).

[37] A. Jablonski, Adv. Colloid Interface Sci. 8, 213 (1977). 